Relations industrielles

Industrial Relations

\title{
Un bureau de placement
}

Jean Gagné

Volume 3, numéro 3, novembre 1947

URI : https://id.erudit.org/iderudit/1023577ar

DOI : https://doi.org/10.7202/1023577ar

Aller au sommaire du numéro

\section{Éditeur(s)}

Département des relations industrielles de l'Université Laval

ISSN

0034-379X (imprimé)

1703-8138 (numérique)

Découvrir la revue

Citer cet article

Gagné, J. (1947). Un bureau de placement. Relations industrielles / Industrial Relations, 3(3), 43-44. https://doi.org/10.7202/1023577ar

Tous droits réservés @ C Département des relations industrielles de l’Université Laval, 1947
Ce document est protégé par la loi sur le droit d'auteur. L'utilisation des services d'Érudit (y compris la reproduction) est assujettie à sa politique d'utilisation que vous pouvez consulter en ligne.

https://apropos.erudit.org/fr/usagers/politique-dutilisation/ 


\title{
UN BUREAU DE PLACEMENT
}

\author{
Bref aperçu de l'organisation d'un bu- \\ reau de placement efficace, dans une \\ importante industrie de la province de \\ Québec.
}

Jean GAGNE

Dans le domaines des relations industrielles, il est un fait bien établi aujourd'hui : c'est que la personne qui désire du travail ne cherche pas seulement une position mais, en réalité, des moyens de subsistance, une sécurité raisonnable, une chance de parvenir à un travail intéressant et d'avenir, pour tout dire, une chance de faire reconnaître ses mérites en termes de salaire et d'avancement. Et si l'on veut s'en occuper, cette personne est souvent prête à devenir un ouvrier de carrière. Or la direction d'une entreprise, si elle est prudente et réellement progressive, préfère employer un ouvrier qui sera non seulement un «numéro sur une liste de paye, » mais un travailleur consciencieux, un partenaire. En effet, une gérance sage ne recherche pas seulement à recruter du personnel mais à mobiliser des personnes qui montrent de l'intelligence, de l'initiative, voire même de l'enthousiasme à réaliser la fin de l'entreprise. Et ceci ne peut être réalisé de nos jours qu'en apportant un soin particulier au choix, à l'embauche, à l'adaptation de l'employé à son nouveau milieu et à son travail afin qu'il réalise sa place dans cette organisation.

Ce sont des raisons comme celles-ci qui amènent les entreprises à organiser un bureau de placement adéquat. Ce sont sans doute là les raisons qui ont présidé à la réorganisation du bureau d'engagement et à l'amélioration de la méthode d'embauchage dans une entreprise de la région de Montréal. Nous ne voulons pas donner cet exemple comme un modèle parfait. Loin de là. Mais malgré leur simplicité, ce plan et cette méthode ont déjà produit d'heureux résultats selon l'opinion des employeurs et des employés.

Voici en quelques mots le processus que les candidats doivent suivre pour l'obtention d'un emploi dans cet établissement et comment on voit à leur adaptation au travail sous la direction du $\mathrm{Bu}$ reau de personnel.

D'abord choix et engagement du nouvel employé. Les candidats attendent dans deux salles d'attente propres et attrayantes : l'une pour les hommes, l'autre pour les femmes. Chacun passe alors par trois bureaux différents où se donnent trois genres d'entrevues. Premièrement, l'entrevue préliminaire. Le but de cette entrevue est d'éliminer les candidats non sérieux ou ceux qui ne se présentent que pour obtenir des renseignements. Ensuite a lieu l'entrevue régulière avec le directeur du personnel qui connaît les positions vacantes. Il offre différentes positions, donne les informations nécessaires, essaie de faire parler le candidat pour le mieux connaître et l'apprécier, le tout de façon très amicale. Si le candidat accepte l'emploi, il passe dans un troisième bureau où l'on remplit les formules nécessaires pour l'engagement. Le nouvel employé (homme ou femme) est alors conduit dans une autre salle d'attente. Ici encore, hommes et femmes sont séparés.

L'avantage de ce procédé, en plus de soigner la première impression de la personne qui se présente au travail, est de permettre au candidat de ne pas être influencé ni troublé soit par les anciens employés ou par les personnes d'un autre sexe ... Il se sent plus à l'aise, a plus de chances de s'exprimer et l'on peut mieux contrôler sa franchise, son sérieux. Ceci a aussi pour but indirect de diminuer le mouvement de la main-d'oeuvre.

Le deuxième stage est celui de la réception du nouvel employé. Des salles d'attente, on conduit les nouveaux employés dans une salle de conférence (Main Gate Hall). On donne alors aux employés une brève conférence sur l'histoire de l'entreprise, sur ses traditions, sa politique, ses règlements et sur la marche générale de la production.

On y donne aussi des informations sur les heures de travail, sur le temps et l'endroit de la paye, le surtemps, le fonds de pension, la sécurité au travail, les règlements d'atelier et ceux qui concernent l'absentéisme et les retards, ainsi que les 
moyens éducationnels et récréatifs qui existent à l'intérieur de l'établissement.

Viennent ensuite quelques conseils concernant le genre d'occupation, les compagnons de travail, les contremaîtres. L'ouvrier peut poser des questions. Après quoi le conférencier l'accompagne jusqu'à l'hôpital où il subit un examen médical. Des messagers de chaque département viennent alors le chercher pour lamener au lieu du travail. Les contremaîtres doivent continuer ce travail de bon accueil avec le nouvel employé. (On a suggéré de retenir les services d'employés sérieux et compétents pour initier les nouveaux employés, les entraîner et les suivre à l'ouvrage. Ce point n'a pas encore été accepté. En effet, l'entraînement des nouveaux ouvriers est trop important pour le laisser entre les mains de n'importe quel irresponsable.) Le dernier stage du processus est de suivre le nouvel employé à son travail au moyen d'entrevues périodiques entre l'ouvrier et le contremaître.

La même personne qui s'est occupée des nouveaux employés à l'entrée principale de l'usine s'occupera d'eux à l'intérieur. Elle rencontrera chacun d'eux au cours de la première semaine de travail, à la troisième semaine, ensuite à la sixième, à la treizième et enfin dans les six mois de leur engagement si nécessaire (entrevues courtes). On garde les dossiers de tout ceci. On prend tous les moyens pour que l'initiation de l'ouvrier au travail ne devienne affaire de routine.

De cette façon, on veut montrer à l'ouvrier qu'on s'intéresse à lui. On sait qu'en principe toute amélioration dans le domaine du personnel doit être nécessairement une étape vers l'établissement de relations plus humaines entre les agents de maîtrise et les ouvriers. Comme on le voit, d'après la méthode suivie, le contremaître est comme obligé de s'occuper de ses employés, de voir si la position attribuée leur convient, de connaître leurs aspirations et de les encourager. Ouvriers et contremaître ne s'en porteront que mieux. La production de même.

Evidemment il existe d'autres méthodes plus compliquées et plus complètes que celle dont on se sert dans cette usine. Elle est certes susceptible d'amélioration. A l'état actuel, elle s'avère cependant pratique et efficace. Ce bureau de placement, la responsabilité de l'embauchage et de la surveillance des ouvriers au travail relèvent du département du personnel, ou plus précisément du directeur du personnel de l'établissement.

S'il y a d'autres entreprises qui ont un tel bureau et voudraient nous faire connaître la méthode qu'elles emploient, nous serions heureux de recevoir toute information sur le sujet.

\section{ASSOCIATION ET CORPORATION}

QUESTION: D'après la Loi des relations ourrières, qu'est-ce qu'on entend par le mot "association" et comment le distinguer du concept de "corporation"? DECISION rendue par la Commission des relations ouvrières de la province de Québec, le 28 mars 1947.

L'Association des Infirmières de la province de Québec, corporation formée des infirmières admises à exercer leur profession dans la province de Québec qui existe légalement depuis le 31 décembre 1946 et le Syndicat des Gardes-Malades de Montréal, formé en vertu des dispositions de la Loi des syndicats professionnels, ont tous deux présenté une requête à la Commission des relations ouvrières pour être accrédités comme agence de négociations auprès de l'Hôpital du Sacré-Coeur de Cartierville.

Chacune des deux parties s'est opposée à la requête de l'autre, l'Association des Infirmières en prétendant que le Syndicat n'était pas dans les conditions prévues par la Loi pour être reconnu, le Syndicat des Gardes-Malades, en affirmant que cette dernière ne devait pas être considérée comme une association au sens de la Loi des relations ouvrieres. 